\section{Controlling Porphyromonas gingivalis requires Vim}

Hundreds of diverse bacterial taxa inhabit the human oral cavity. Over evolutionary time, potential pathogens, such as Porphyromonas gingivalis, have become host-adapted and typically maintain a clinically silent status. For P. gingivalis, this involves walking a regulatory tightrope; colonization and nutrient acquisition must be maintained without encroaching on the host or inducing tissue destruction. Colonization depends on fimbriae and outer-membrane proteins with specificity for the hard and soft tissues in the mouth. However, these proteins can also disrupt cytokine networks and innate immunity, and trigger mistimed and misdirected signalling in host cells. As an asaccharolytic organism, $P$. gingivalis relies on peptides for all carbon and nitrogen needs. To facilitate peptide availability, $P$. gingivalis produces a suite of at least 10 proteases, the most potent of which are the arginine (RgpA, RgpB) and lysine (Kgp) -specific gingipains. If there is too much protease production periodontal tissues degrade, too little and the organism starves. The dilemma, then, for $P$. gingivalis is to achieve the flexibility to colonize and accumulate on available surfaces without causing tissue damage either directly through protease action or indirectly through dysfunctional host immunity. A complex regulatory network must underlie this adaptability, probably involving transcriptional and posttranscriptional mechanisms, but until recently, few components of this circuitry, especially at the post-translational level, were known.

Enter VimA. In this issue, Osbourne et al. (2010) show that the VimA protein can control capsular biogenesis and fimbrial synthesis. A vimA mutant strain displayed an irregular polysaccharide capsule and more fimbriae compared with the parental strain. In addition, loss of VimA impacted glycosylation of outer- membrane proteins and acetylation of an extracellular protein. In all, as observed by mass spectrometry of the outer membrane fraction of the vimA mutant, 20 proteins were aberrantly expressed and nine proteins were missing, indicating that VimA plays a role in the glycosylation and anchorage of surface proteins. These results are consistent with previous reports from this group (Vanterpool et al., 2006) showing that VimA also regulates gingipain activity, as there is a late onset of gingipain activity in the vimA mutant. Kgp and Rgp activity in exponential growth phase is $80 \%$ less than that of the wild-type. In stationary phase, gingipain activity is $40-50 \%$ less than that of the wild-type. Furthermore, there is no membrane-associated RgpA or Kgp in the absence of VimA, which suggests that VimA is involved in the process of anchoring gingipains in the membrane.

VimA thus occupies a node of a regulon that controls major surface structures and proteases at the post-translational level. What kind of protein is VimA? In silico analysis of VimA predicts that this protein belongs to the acyl-CoA $N$-acyl superfamily (FemXAB nonribosomal peptidyltransferase subfamily) and/or has sortase-like activity. These enzymes are predicted to have biosynthetic and biogenic properties that extend beyond peptidoglycan metabolism. This would allow a tentative prediction that VimA can perform linking reactions that anchor proteins in the membrane and attach carbohydrates and other groups to proteins. Intriguingly these FemXAB enzymes have hitherto been functionally defined in Gram-positives, and VimA may be the founding member of a novel posttranslational control mechanism in Gramnegatives. Of additional interest is the physical arrangement of vimA downstream of $\operatorname{rec} A$ and part of a multi-gene transcriptional unit. Why is VimA tied to RecA? Part of the answer may be that VimA is also involved in oxidative stress responses (Johnson et al., 2004) and an operon arrangement may be an effective strategy to coordinate responses to stress in the periodontal pocket. Moreover, in Escherichia coli, the RecA-DNA filament is an allosteric effector of LexA autoproteolysis, and LexA is a repressor of the SOS response (Little \& Mount, 1982). It will be interesting to determine if RecA in $P$. gingivalis has an effect on the stability of VimA. Downstream of vimA are vimE and $v i m F$, which also independently affect protease activity. Clearly, $P$. gingivalis devotes a significant proportion of its genome to protease production and regulation.

Another aspect of this regulatory system to be explored is the integration of VimA with mechanisms that control protease and fimbrial expression at the transcriptional level. $P$. gingivalis contains a modest number of two-component systems (TCSs); only six are predicted in one of the sequenced strains. However, the hybrid TCS GppX regulates protease activity (Hasegawa et al., 2003), and the FimS/ FimR TCS controls production of both types of $P$. gingivalis fimbriae.

Furthermore, Fims may interact with other sensor kinases and can control the transcription of around $10 \%$ of the genome (Lo et al., 2010). Gingipain
Microbiology Comment provides a forum for discussion of scientific issues arising directly from papers published in the journal. The authors of papers under discussion will be offered an opportunity to respond.

Guidelines on how to submit a Microbiology Comment article can be found in the Instructions for Authors at http://mic.sgmjournals.org

It should be noted that the Editors of Microbiology do not necessarily agree with the views expressed in Microbiology Comment.

A. Fouet, Editor-in-Chief 
activity and exopolysaccharide production are also controlled through tyrosine phosphorylation/dephosphorylation pathways (Maeda et al., 2008). And in the 'if you're not confused, then you don't understand' category, gingipains are also required for processing of the long fimbrial subunit (Onoe et al., 1995) and can regulate fimA mRNA levels (Xie et al., 2000). Further study of VimA will undoubtedly reveal more important circuitry. It is also worthy of note that Osbourne et al. (2010) showed that the VimA mutant is more sensitive to globomycin and vancomycin, making VimA function a potential target for therapeutic intervention.

\section{Richard J. Lamont}

Department of Oral Biology, University of Florida, Gainesville, FL 32607, USA

Correspondence: Richard J. Lamont (rlamont@dental.ufl.edu)

\section{REFERENCES}

Hasegawa, Y., Nishiyama, S., Nishikawa, K., Kadowaki, T., Yamamoto, K., Noguchi, T. \& Yoshimura, F. (2003). A novel type of twocomponent regulatory system affecting gingipains in Porphyromonas gingivalis. Microbiol Immunol 47, 849-858.

Johnson, N. A., McKenzie, R., McLean, L., Sowers, L. C. \& Fletcher, H. M. (2004). 8-oxo7,8-dihydroguanine is removed by a nucleotide excision repair-like mechanism in

Porphyromonas gingivalis W83. J Bacteriol 186, 7697-7703.

Little, J. W. \& Mount, D. W. (1982). The SOS regulatory system of Escherichia coli. Cell 29, 1122.

Lo, A., Seers, C., Dashper, S., Butler, C., Walker, G., Walsh, K., Catmull, D., Hoffmann, B., Cleal, S. \& other authors (2010). FimR and FimS: biofilm formation and gene expression in Porphyromonas gingivalis. J Bacteriol 192, 1332-1343.

Maeda, K., Tribble, G. D., Tucker, C. M., Anaya, C., Shizukuishi, S., Lewis, J. P., Demuth, D. R. \& Lamont, R. J. (2008). A Porphyromonas gingivalis tyrosine phosphatase is a multifunctional regulator of virulence attributes. Mol Microbiol 69, 1153-1164.

Onoe, T., Hoover, C. I., Nakayama, K., Ideka, T. Nakamura, H. \& Yoshimura, F. (1995).

Identification of Porphyromonas gingivalis prefimbrilin possessing a long leader peptide: possible involvement of trypsin-like protease in fimbrilin maturation. Microb Pathog 19, 351364.

Osbourne, D. O., Aruni, W., Roy, F., Perry, C., Sandberg, L., Muthiah, A. \& Fletcher, H. M. (2010). Role of vimA in cell surface biogenesis in Porphyromonas gingivalis. Microbiology 156, 2180-2193.

Vanterpool, E., Roy, F., Zhan, W., Sheets, S. M., Sangberg, L. \& Fletcher, H. M. (2006). VimA is part of the maturation pathway for the major gingipains of Porphyromonas gingivalis W83. Microbiology 152, 33833389 .

Xie, H., Chung, W. O., Park, Y. \& Lamont, R. J. (2000). Regulation of the Porphyromonas gingivalis fimA (Fimbrillin) gene. Infect Immun 68, 6574-6579.

DOI 10.1099/mic.0.041251-0 